\title{
The effect of geometry on the adhesive behavior of bio-inspired fibrils
}

\author{
Zhilong Peng and Shaohua Chen* \\ Received 25th March 2012, Accepted 24th July 2012 \\ DOI: $10.1039 / \mathrm{c} 2 \mathrm{sm} 26390 \mathrm{~d}$
}

Inspired by the special climbing ability of geckos and insects, the effects of geometry, including the endshape and size, on the adhesion features of bio-inspired fibrils, are investigated. It is found that, the adhesion force of a mushroom-shaped fibril in perfect contact with a rigid substrate decreases with the increase of the peeling angle, but it will increase with an increasing flange thickness at a determined peeling angle. With the same contact length, the mushroom-shaped fibril can achieve much larger adhesion forces than the cylindrical or spatular one due to a larger effective region of the cohesive zone. The effect of the shaft width of the mushroomed-shaped fibril on the adhesion force with and without interfacial defects is also considered, and the results are further compared qualitatively with the experimental ones. A critical contact length for the mushroom-shaped fibril is further found, above which the adhesion force attains a maximum. For a multi-fibril structure, the phenomenon of almost equal load sharing for each fibril is verified numerically, which is consistent with the existing experimental observation. All the results in this paper should be helpful for the understanding of the micro-adhesion mechanism of biological adhesive systems and the design of novel adhesives.

\section{Introduction}

The amazing adhesive ability of geckos to climb and detach from ceilings and vertical walls at will has been studied extensively since the micro-mechanism of the attachment was shown to be predominantly due to intermolecular surface forces., ${ }^{1,2}$ Experiments have found that many small hairs, as the lowest level, cover the surface of the hierarchical adhesive system on geckos feet, which not only allow a large contact area on almost any surface but also accumulate the micro-intermolecular forces leading to high adhesion forces. It is well known that the geometries of contact elements are extremely important factors affecting adhesion, and the size and shape of the small hairs vary with the weight of the insects and animals. ${ }^{3-9}$ Comparing different biological adhesive systems, it is found that, as the lowest level of the hierarchical adhesive system, mushroomshaped fibrils or spatula-shaped fibrils are often adopted as the main contact elements by many insects in nature. ${ }^{8,10}$ Del Campo et $a .^{6}{ }^{6}$ studied experimentally the effect of contact shape and found that the contact geometry could exert a strong effect on the adhesion. High adhesion could be obtained by a mushroom-like or spatular terminal. In order to explain the experimental observations, ${ }^{6}$ Spuskanyuk et al. ${ }^{5}$ analyzed numerically the adhesion of a mushroom-shaped fibril and a flat punch in contact with a rigid substrate. They found that the better adhesion of the mushroom-shaped fibril was due to the compressive stress at the

LNM, Institute of Mechanics, Chinese Academy of Sciences, Beijing, 100190, China. E-mail: chenshaohua72@hotmail.com; Fax: +86 10 82543977; Tel: +861082543960 periphery of the flange, which could prevent defects from propagating. ${ }^{5}$ Inspired by the attachment system of beetles from the Chrysomelidae family, mushroom-shaped fibrillar structures were characterized by a variety of techniques and compared with flat surface structures made of the same material. ${ }^{8}$ It was found that the pull-off force and peel-off strength of the structured specimen were more than two times that of the flat specimen. The other adhesive characteristics of a mushroom-shaped fibril, such as the shear effect on adhesion, suction components and dynamic adhesive behaviors, have also been studied systematically and experimentally. ${ }^{11-14}$ Unlike the spatula-shaped contact element that requires active lateral loading for proper functioning ${ }^{1,10}$ and is associated with a muscle-driven temporary attachment involved in locomotion, ${ }^{15}$ the mushroom-shaped contact element is a passive attachment device, which is able to hold for a virtually unlimited period of time with no muscular mechanisms being invoked and can be used in a long-term process of pairing. ${ }^{12,16}$ Although recent experiments demonstrated that the mushroom-shaped fibril exhibited exceptionally high adhesion forces, the underlying adhesion mechanism and the effect of geometrical parameters on adhesion are still poorly understood. Inspired by Gao and Chen, ${ }^{17}$ we found a critical contact length for a bio-inspired nano-film, ${ }^{18}$ above which the adhesion force of the film on a substrate attained a maximum. Is there a similar length scale that controls the adhesion of a mushroom-shaped fibril?

On the other hand, geckos use a spatula-shaped fibril as the smallest contact element, which is similar to a nano-film with a finite length. The micro-adhesion mechanism of a single spatula has been studied by many theoretical models. ${ }^{10,18-20}$ Tian et al. ${ }^{10}$ 
proposed a frictional adhesion model and found that the peel-off force varied strongly with the peeling angle. A bio-inspired nanofilm with a finite contact length was investigated by Peng et al., ${ }^{18}$ in which the effects of the contact length, the film thickness and the peeling angles on the adhesion force were considered. The influence of the surface roughness ${ }^{21}$ and the environment relative humidity $^{22}$ on the adhesion of a nano-film were also studied. However, as we know, there are hundreds of thousands of setae on geckos feet and each seta branches into several hundreds of spatulae. Experiment has found that each spatula almost supports equal loads when a gecko attaches to a surface. ${ }^{23}$

In order to obtain a deeper understanding of the microadhesion mechanisms in biology, adhesive characteristics of a mushroom-shaped fibril, a spatula-shaped fibril and a multi-fibril structure are investigated in this paper numerically and theoretically. The effects of the shape and size of the structures are mainly focused on. Comparisons of our results and existing experimental ones are given and discussed. The results in this paper should be helpful for the understanding of the adhesion mechanisms of some insects and animals in nature, and the design of novel biomimetic adhesive materials or structures.

\section{The adhesion of a mushroom-shaped fibril and a spatular fibril}

Only a few studies based on fracture mechanics have been carried out to investigate the adhesion mechanisms of mushroom-shaped fibrils. ${ }^{5,24,25}$ In this section, a plane strain model is established as shown in Fig. 1, in which a mushroom-shaped fibril contacts adhesively with a rigid substrate with flange thickness $h$, shaft width $d$, adhesive contact length $L$ and shaft angle $\theta$. A peeling force $P$ acts at the end of the fibril along the shaft direction.

\subsection{Numerical analysis}

Numerical simulation is carried out based on the code developed by Dr. Patrick Klein of Sandia National Laboratory (www.tahoe.sourceforge.net). The fibril is assumed to be elastic with Young's modulus $E=2 \mathrm{GPa}$. 4-Noded plane strain elements modified by Simo et al. ${ }^{26}$ are chosen in order to improve the performance of bending and the nearly incompressible state of deformation. Molecular interactions between the two contact

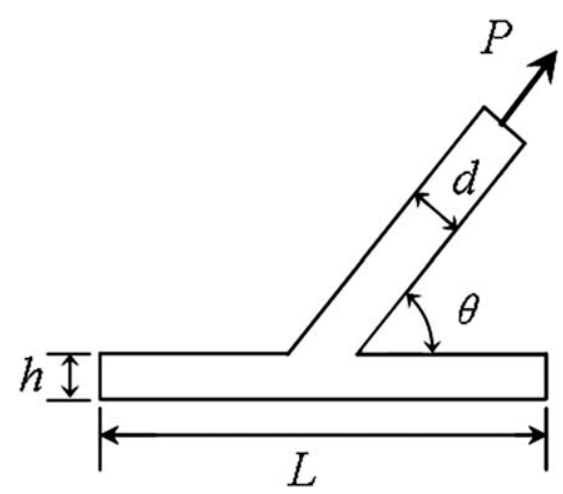

Fig. 1 Schematic of a mushroom-shaped element in contact with a rigid substrate with adhesive length $L$, flange thickness $h$, shaft width $d$ and peeling force $P$. surfaces are represented by a layer of cohesive surface elements. The constitutive relation of the cohesive surface element was developed by Tvergaard and Hutchinson. ${ }^{27}$ Other cohesive models ${ }^{28-31}$ could in principle be used to model the molecular adhesion too. The Tvergaard-Hutchinson model is chosen in the present study because it preserves the van der Waals energy regardless of the pulling direction. Whereas, other cohesive models with various considerations of tension versus shear dominated separation do not necessarily preserve the interaction energy. ${ }^{32}$

The detailed relationship of the traction-separation in the Tvergaard-Hutchinson model can be found in ref. 18 and 27. The material constants for geckos are taken from Gao et al. ${ }^{32}$ as follows,

$$
\left\{\begin{array}{l}
\sigma_{0}=20 \mathrm{MPa}, \quad \Delta \gamma=0.01 \mathrm{~J} \mathrm{~m}^{-2} \\
\delta_{\mathrm{n}}^{\mathrm{c}}=\delta_{\mathrm{t}}^{\mathrm{c}}=0.5 \mathrm{~nm}, \quad E=2 \mathrm{GPa}
\end{array}\right.
$$

where $\sigma_{0}$ is the interface theoretical strength, $\Delta \gamma$ is the adhesion energy, $\delta_{\mathrm{n}}^{\mathrm{c}}$ and $\delta_{\mathrm{t}}^{\mathrm{c}}$ are the maximum effective interaction distances in the normal and tangential directions of the interface, respectively. $E$ is the Young's modulus of the mushroom-shaped adhesive material.

In previous experiments, mushroom-shaped fibrils were often fabricated with a peeling angle $\theta=90^{\circ}$ to study the adhesion behaviors and to compare the adhesion force with that of other contact shapes. ${ }^{6,11-13,33}$ Theoretical models for the adhesion of a mushroom-shaped fibril with $\theta=90^{\circ}$ were established to attempt to explain the experimental observations. ${ }^{5,25}$ However, the geometrical effects with various parameters of the mushroomshaped fibril on the adhesion force are not clear so far, which will be investigated systematically in this paper to explore the superior adhesion mechanism of such a structure.

Fig. 2(a) shows the adhesion force as a function of the shaft (peeling) angle $\theta$ for a mushroom-shaped fibril with different shaft widths $d$, but with a fixed flange thickness $h=5 \mathrm{~nm}$ and contact length $L=100 \mathrm{~nm}$. The corresponding result for a spatular fibril is also given in Fig. 2(a) for comparison, in which the spatula has the same contact length and film thickness as the mushroom-shaped fibril. It is shown that the adhesion force of the mushroom-shaped fibril decreases with the increase of the peeling angle $\theta$, but it increases with the increase of the shaft width $d$ at a given $\theta$. Comparing the adhesion force of the mushroom-shaped fibril with that of the spatular one, one can see that the adhesion force of the former is much larger than that of the latter at the same peeling angle, which is consistent with the experimental observations. ${ }^{6}$

Fig. 2(b) gives the adhesion force as a function of the peeling (shaft) angle $\theta$ for the mushroom-shaped fibril with a determined shaft width and a determined contact length, but with different flange thicknesses $h$. A similar varying tendency to that in Fig. 2(a) can be found, that is the adhesion force decreases with the increase of the peeling angle $\theta$ when the thickness of the flange is fixed, but it increases with an increasing flange thickness at the same peeling angle. One may note the results in ref. 21 , where the Lennard-Jones potential was used to calculate the normal adhesion force between a nano-film and a rigid substrate. It was found that the normal interfacial adhesion force would stay constant when the film thickness was above a critical value, 

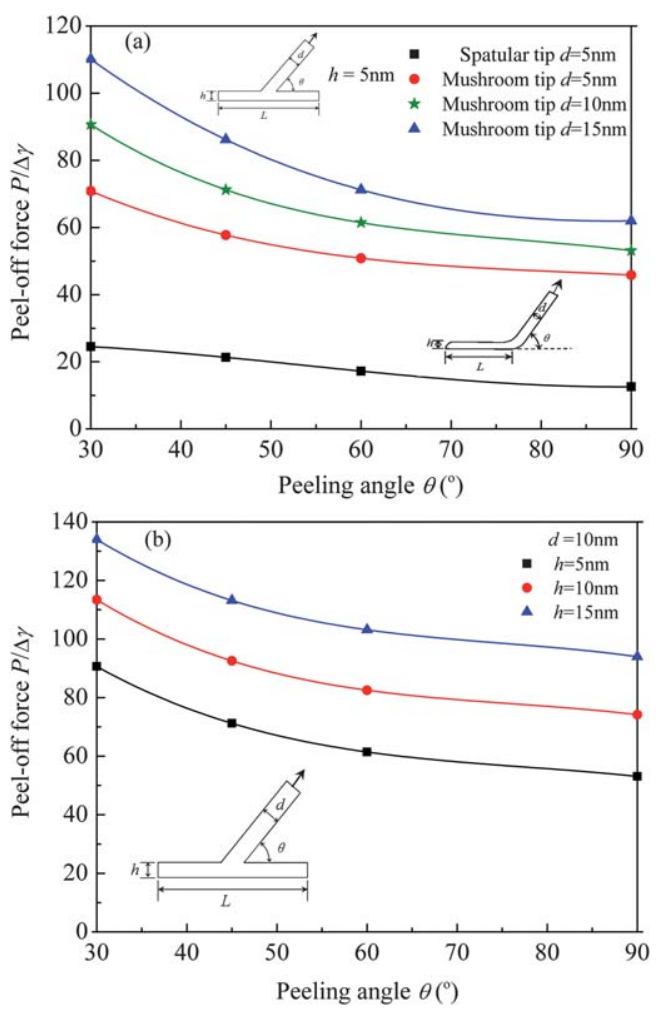

Fig. 2 The non-dimensional peel-off force $P / \Delta \gamma$ as a function of the peeling angle $\theta$ for the mushroom-shaped element in contact with a rigid substrate, and a case with a spatular fibril is also given for comparison. (a) For different shaft width $d$ and a fixed flange thickness $h$. (b) For different flange thicknesses $h$ and a fixed shaft width $d$.

about $5 \mathrm{~nm}$. This was because no external load was considered in ref. 21, which could not result in bending and stretching strain energies in the nano-film and only the van der Waals interaction energy was included. While in the present paper, under a peeling force, the bending and stretching strain energies will be no doubt be produced and stored in the flange. Furthermore, the thicker the flange, the more bending energy will be needed until the fibril is detached from the substrate.

One should note that, in many experiments, ${ }^{6,33}$ a mushroomshaped fibril with a small shaft width exhibits better adhesion than that with a larger one. At first glance, the experimental observations contradict our results in Fig. 2(a), where the adhesion force increases with the increase of the shaft width. What is the reason? Actually, the interface in the present model is assumed to be perfect without any defect in the contact region, while interfacial defects cannot be avoided in a real experiment. According to the theoretical analyses of Gao et al. ${ }^{32}$ and Hui et al. ${ }^{34}$ the adhesion strength can reach the theoretical one for a flat punch in frictionless and perfect contact with a smooth rigid substrate. Here, two problems are introduced, one is the effect of the interfacial defects in the contact region on the adhesion force and the other is that of interfacial friction.

In fact, Spuskanyuk et al..$^{5}$ compared the interfacial stress distributions of a mushroom-shaped fibril and a flat punch adhering on a flat substrate numerically, and found that when the shaft width was much smaller than the contact length, the contact area under the shaft supported a tensile stress, but near the periphery of the flange, the stress was very low and even slightly compressive. Therefore, any defect (interfacial defects inevitably exist in experiments) confined to the compressive region will not propagate, which results in better adhesion. With the increase of the shaft width, all the interfacial tractions tend to be tensile, especially when the shaft width approaches the length of the flange (the mushroom-shaped fibril becomes a flat punch at this time). Thus, defects near the contact edges would experience a driving force for propagation, which leads to poor adhesion. The conclusions are verified in Fig. 3. Fig. 3(a) exhibits the adhesion force of a mushroom-shaped element in frictionless contact with a rigid substrate as a function of the shaft width with and without edge defects. One can see that, in the case without edge defects, the adhesion force increases with an increasing shaft width and reaches theoretical strength when the mushroom-shaped element becomes a flat punch one, which is consistent with the analysis of Gao et al. ${ }^{32}$ and Hui et al. ${ }^{34}$ While in the case with edge defects and the mushroom-shaped fibril is in frictional contact with a substrate (Fig. 3(b)), the adhesion force of the mushroom-shaped element increases first and then decreases with the increase of the shaft width. These results qualitatively agree with the experimental observations. ${ }^{6,33}$

Fig. 4 shows the adhesion force of a symmetrical mushroomshaped element and an asymmetrical one, respectively, as a function of the contact length with the peeling angle $\theta=90^{\circ}$. Both cases have the same flange thickness and shaft width $h=d$ $=5 \mathrm{~nm}$. One can see that the adhesion force increases with an increasing contact length, and then attains a saturated value at a
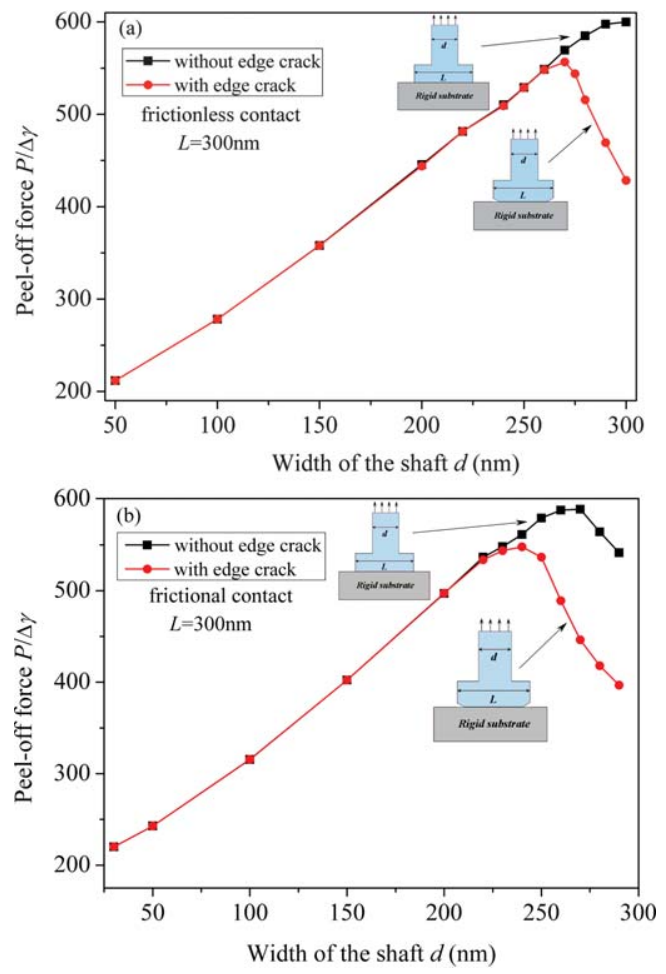

Fig. 3 The non-dimensional peel-off force as a function of the shaft width for a mushroom-shaped element contacting a rigid substrate with and without edge defects, where the contact length is $L=300 \mathrm{~nm}$, the flange thickness is $h=50 \mathrm{~nm}$ and the shaft angle is $\theta=90^{\circ}$. Two cases are considered: (a) frictionless contact, (b) frictional contact. 


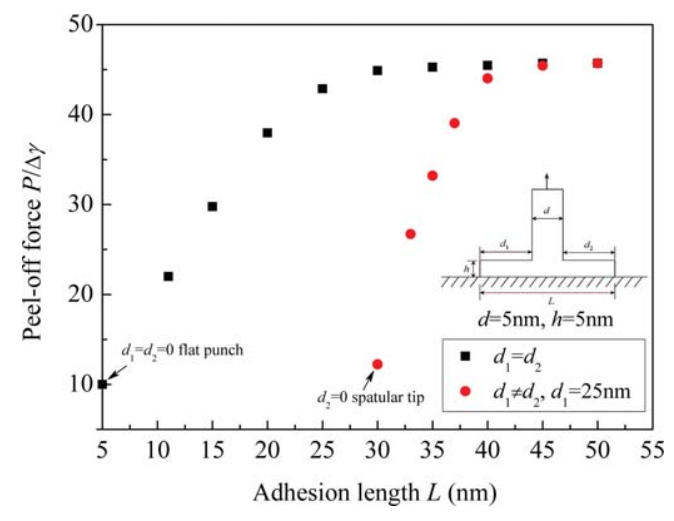

Fig. 4 The non-dimensional peel-off force as a function of the adhesion length for the symmetrical and asymmetrical mushroom-shaped fibrils with a shaft angle of $\theta=90^{\circ}$. The symmetrical fibril can be reduced to a flat punch when $d_{1}=d_{2}=0$, while the asymmetrical one can be reduced to a spatular fibril when $d_{2}=0$.

critical value in both the symmetrical and asymmetric cases. Furthermore, the saturated adhesion forces are equal for both cases but with different critical contact lengths. From Fig. 4, one can also find that the mushroom-shaped element can be reduced to a flat punch element when $d_{1}$ and $d_{2}$ vanish in the symmetric case, while it can be reduced to a spatular pad when $d_{2}=0$ in the asymmetric case. All the above results are consistent with the experimental observations ${ }^{6}$ that the contact shape has a strong effect on the adhesion force and the mushroom-shaped contact element shows an exceptionally high adhesion. As for the case of $\theta \neq 90^{\circ}$, the mushroom-shaped element is obviously asymmetric no matter whether $d_{1}=d_{2}$ or $d_{1} \neq d_{2}$. However, it can be inferred that critical values of $d_{1}$ and $d_{2}$ should also exist. Due to the complexity of the different combinations of $d_{1}$ and $d_{2}$, detailed discussions are neglected in the present paper.

\subsection{Theoretical analysis}

Similar to the technique adopted in ref. 18, a theoretical model is established in Fig. 5(a) in order to predict the effective contact length (i.e. the cohesive zone length) of the mushroom-shaped element in the case of $\theta=90^{\circ}$. Assuming the radius of the cohesive zone is two times that of the flange thickness. ${ }^{10}$ The relation among the strain energy, the work done by the peeling force and the adhesion energy that should be overcome, can be expressed as

$$
2 \int_{0}^{l} \sigma_{0} \delta_{\mathrm{n}}^{\mathrm{c}} \frac{R-\sqrt{R^{2}-x^{2}}}{R} \mathrm{~d} x=\frac{1}{2} p d \delta_{\mathrm{n}}^{\mathrm{c}}-E \varepsilon^{2} L_{\mathrm{R}} h
$$

where $l$ is half of the effective contact length, $p$ is the distributed load exerted on the flange by the shaft, $p=P / d$. $\varepsilon$ is the elastic strain, $L_{\mathrm{R}}$ is the arc length which is denoted as $L_{\mathrm{R}}=R \theta$. From eqn (2), the effective contact length $l$ can be found from the following relationship

$2 \Delta \gamma\left\{l-\frac{R}{2}\left[\arcsin \frac{l}{R}+\frac{1}{2} \sin \left(2 \arcsin \frac{l}{R}\right)\right]\right\}=\frac{1}{2} p d \delta_{\mathrm{n}}^{\mathrm{c}}-E \varepsilon^{2} L_{\mathrm{R}} h$

The adhesion force predicted by the theoretical model and numerical calculation as a function of the contact length is shown
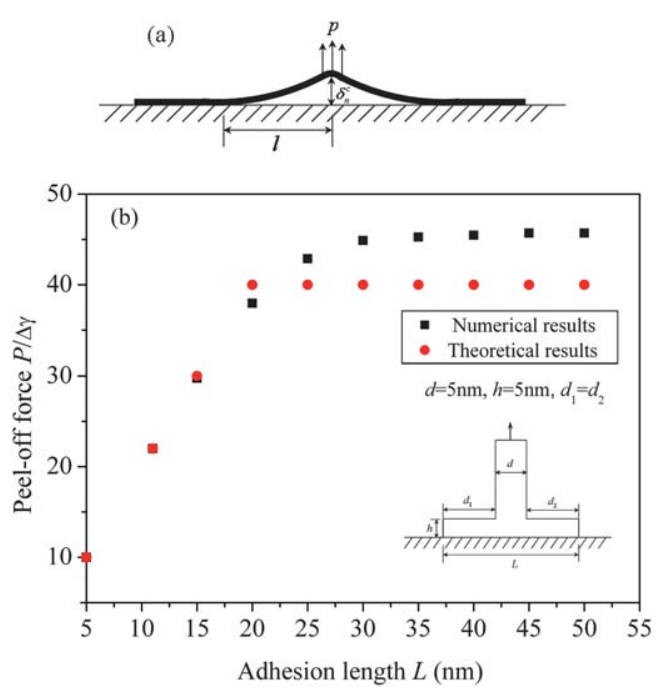

Fig. 5 Theoretical analysis of the adhesion force of a mushroom-shaped fibril in contact with a rigid substrate. (a) The theoretical model with a cohesive zone $2 l$ and a shaft angle of $\theta=90^{\circ} . p$ is an uniform load obtained as $p=P / d$. (b) Comparison of the non-dimensional adhesion force predicted by numerical calculation and theoretical analysis as a function of the adhesion length.

in Fig. 5(b). From Fig. 5(b), one can see that the variation tendency predicted by the simple theoretical model is consistent with that calculated by the numerical one, though the maximum force predicted numerically is slightly higher than the theoretical one. Several reasons could explain the deviation. In the theoretical model, we adopt the assumption that the critical contact length represents the effective one of the cohesive zone. In fact, the cohesive zone consists of not only the one in which the interfacial traction attains the theoretical strength, but also the part in which the interfacial traction is less than the theoretical strength. Second, a circular shape of the cohesive zone is assumed and the bending energy is not included in the total energy balance.

To identify the benefit of a mushroom-shaped or a spatulashaped adhesive fibril in contrast to a cylindrical one and why the mushroom-shaped fibril's adhesion force is much larger than that of the spatula-shaped one, the normal tractions at the contact interface are plotted as a function of the contact length in Fig. 6(a) and (b). Fig. 6(a) shows the distribution of the normal tractions for the mushroom-shaped fibril, while Fig. 6(b) is for the spatula-shaped fibril. It is easy to find that the normal tractions within the cohesive zone are tensile. However, if the contact length is larger than the effective contact length, compressive stresses will emerge ahead of the cohesive zone for both the mushroom-shaped and the spatula-shaped fibrils, which is similar to the analysis of Spuskanyuk et al. ${ }^{5}$ Fig. 6(a) and (b) also exhibit this when the total contact length of the fibrillar pad is smaller than the critical adhesive length, the tractions can attain the interfacial theoretical strength $\sigma_{0}$ uniformly at the moment of peel-off. Comparing the region of the cohesive zone in the mushroom-shaped fibril and the spatula-shaped one with the same contact length, one can see that the length of the cohesive zone subjected to tensile tractions in the mushroom-shaped fibril model is much larger than that in the spatula-shaped one. As a 

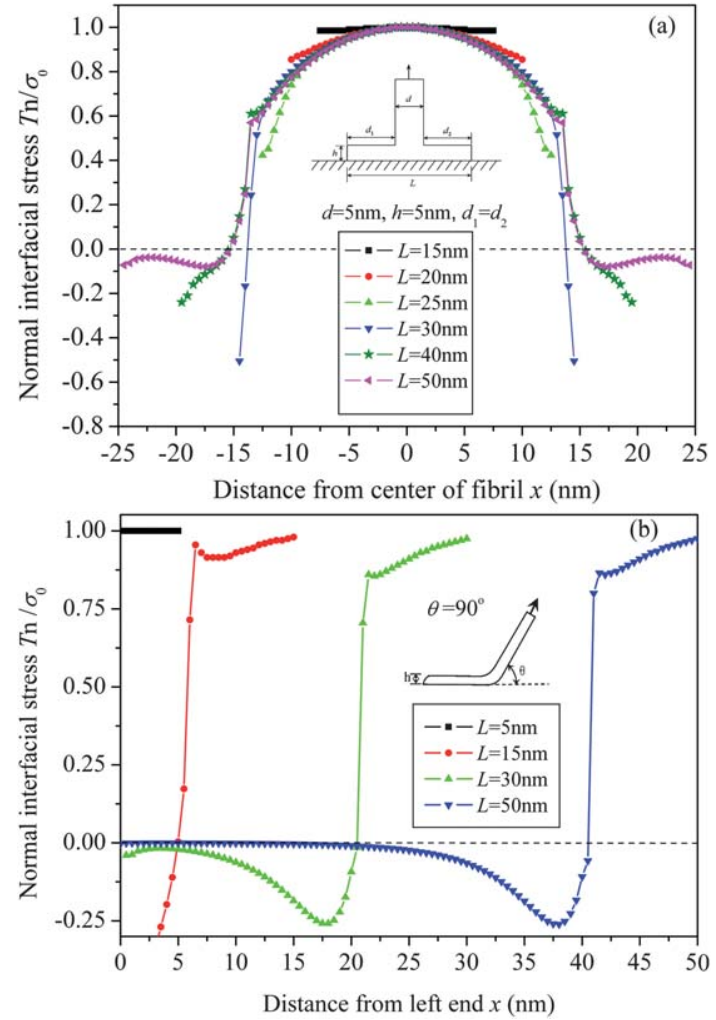

Fig. 6 The distribution of the normalized interfacial normal stress varying as a function of the contact length at the moment of peel-off for cases with different contact lengths. (a) The mushroom-shaped fibril adhering on a flat substrate with a shaft angle $\theta=90^{\circ}$. (b) The spatulashaped fibril adhering on a flat substrate with a peeling angle of $\theta=90^{\circ}$.

consequence, the mushroom-shaped fibril can support a larger adhesion force than that of the spatula-shaped fibril, which could explain the experimental observations ${ }^{6}$ and numerical results in the present paper.

Although some adhesive behaviors of the mushroom-shaped fibril are similar to that of the spatular pad, such as the adhesion force decreasing with the increase of the peeling angle $\theta$, many insects and animals, taking the spatular pads as the smallest contact elements, can control the action of different muscles, such as digital gripping and hyperextension, to switch between attachment and detachment by changing the peeling angle. The mushroom-shaped fibrils that have evolved in nature or artificial fabrications often have a constant shaft angle and the most
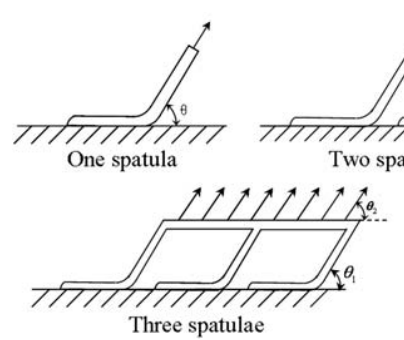

Fig. 7 Schematics of structures with one-, two- and three-spatulae adhering on a rigid substrate.
Table 1 Adhesion forces of structures with one-, two- and three-spatulae adhering on a flat substrate under a peeling force at different peeling angles

\begin{tabular}{|c|c|c|c|c|}
\hline & \multicolumn{4}{|c|}{ Unit: $\mathrm{nN} \mathrm{nm}{ }^{-1}$} \\
\hline & \multicolumn{2}{|c|}{$\theta_{1}=30^{\circ}$} & \multicolumn{2}{|c|}{$\theta_{1}=60^{\circ}$} \\
\hline & $\theta_{2}=30^{\circ}$ & $\theta_{2}=90^{\circ}$ & $\theta_{2}=60^{\circ}$ & $\theta_{2}=90^{\circ}$ \\
\hline One spatula & 0.205 & & 0.111 & \\
\hline Two spatulae & 0.4215 & 0.0458 & 0.224 & 0.0706 \\
\hline Three spatulae & 0.6347 & 0.0721 & 0.3373 & 0.108 \\
\hline
\end{tabular}

commonly used angle is $\theta=90^{\circ}$. As discussed above, the mushroom-shaped fibrils are passive attachment devices with no muscular mechanisms being invoked. Why some animals adopt the mushroom-shaped fibril as the smallest contact element needs further experimental investigation.

\section{The adhesion feature of a multi-fibril structure}

As we know there are hundreds of thousands of setae on gecko feet and each seta branches into several hundreds of spatulae. Experimental measurements have shown that each spatula at the end of a gecko's seta almost support equal peeling force, ${ }^{23,34}$ which is often called equal load sharing (ELS). The concept of ELS has also been adopted to analyze the effect of contact splitting on adhesion. ${ }^{3,7}$ In this part, the adhesive behaviors of structures with one-, two- and three-spatulae are investigated. Considering the rolling-in and rolling-out behaviors of a gecko's foot, the clockwise moment and counter-clockwise moment exerted at the end of the seta can lead to peeling forces in different directions, which control the attachment and detachment behaviors of each spatula. ${ }^{32}$ Therefore, two kinds of loads are considered in our model, one is along the spatula shaft, i.e., $\theta_{2}$ $=\theta_{1}$, and the other is vertical, i.e., $\theta_{2}=90^{\circ}$ as shown in Fig. 7.

The adhesion forces of structures with one-, two- and threespatulae adhering on a rigid substrate are calculated and given in Table 1 , in which we take $\theta_{1}=30^{\circ}$ and $\theta_{1}=60^{\circ}$, respectively. From Table 1, we find that the adhesion forces of structures with two- and three-spatulae approximately equal two and three times that of the single spatula when $\theta_{2}=\theta_{1}$. The results agree well with the theoretical ones, ${ }^{7,35}$ where the effect of contact splitting on the adhesion of thin-film-ended surface elements with a constant contact area was analyzed ${ }^{7}$ and discussed. ${ }^{35}$ Furthermore, a much larger adhesion force of the spatula array with $\theta_{2}=\theta_{1}$ than that with $\theta_{2}=90^{\circ}$ also agrees with the results obtained by Gao et al., ${ }^{32}$ where finite element calculations found the attachment force of a curved seta pulling at $30^{\circ}$ to be approximately an order of magnitude larger than that at $90^{\circ}$.

\section{Conclusions}

The adhesion features of a single fibril and a multi-fibril structure are investigated in this paper. The effects of the geometrical parameters of a mushroom-shaped fibril on the adhesion are analyzed systematically. It is found that the adhesion force of the mushroom-shaped element decreases with the increase of the 
peeling angle, while it increases with the increasing flange thickness at a given peeling angle. The adhesion force of the mushroom-shaped fibril in contact with a substrate as a function of the shaft width with and without interfacial defects is also analyzed for two cases: frictional contact and frictionless contact. It is found that the adhesion force increases with the increase of the shaft width for the perfectly frictionless contact, while the adhesion force increases first and then decreases with the increase of the shaft width for the defectively frictional contact, which is consistent with the experimental results. ${ }^{33}$ Comparing the mushroom-shaped element with other shaped ones, the former has a significantly higher adhesion than the latter, which is due to a larger cohesive region and compressive interfacial tractions at the periphery of the flange that can prevent crack-like defects from propagating. The findings agree well with the experimental observations. ${ }^{6,8}$ Similar to the adhesion of the spatula, a critical contact length for the mushroom-shaped fibril is found, above which the adhesion force attains a maximum. However, reversible adhesion mechanisms of the mushroom-shaped contact element need further study in future work. In addition, the feature of "equal load sharing" of a spatula array found in experiment is also proved by the numerical calculations. The results in the present paper should be helpful not only for the understanding of some bioexperimental phenomena, but also for the design of novel adhesives.

\section{Nomenclature}

\begin{tabular}{ll}
\hline$\sigma_{0}$ & Interfacial theoretical strength \\
$\Delta \gamma$ & Adhesion energy \\
$\delta_{\mathrm{n}}^{\mathrm{c}}$ & Effective interaction distance in the normal \\
& direction of the interface \\
$\delta_{\mathrm{t}}^{\mathrm{c}}$ & Effective interaction distance in the tangential \\
$E$ & direction of the interface \\
$P$ & Young's modulus of the fibril \\
$L$ & Peeling force acting at the end of the shaft \\
$\theta$ & Contact length between the fibril and the \\
$\theta_{1}$ & substrate \\
$\theta_{2}$ & Peeling angle of the mushroom-shaped fibril \\
$d_{1}$ & Peeling angle of the spatular fibril \\
$d_{1}$ & Direction of the peeling force acting on the \\
$d_{2}$ & multi-fibril structure \\
$L_{\mathrm{R}}$ & Shaft width of the mushroom-shaped fibril \\
$h$ & Contact length on the left side of the \\
$p$ & mushroom-shaped fibril \\
& Contact length on the right side of the \\
& mushroom-shaped fibril
\end{tabular}

\section{Acknowledgements}

The work reported here is supported by NSFC through grants \#10972220, \#11125211, \#11021262 and the 973 Nano-Project (2012CB937500).

\section{References}

1 K. Autumn, Y. A. Liang, S. T. Hsieh, W. Zesch, W. P. Chan, T. W. Kenny, R. Fearing and R. J. Full, Nature, 2000, 405, 681-685.

2 K. Autumn, M. Sitti, Y. C. A. Liang, A. M. Peattie, W. R. Hansen, S. Sponberg, T. W. Kenny, R. Fearing, J. N. Israelachvili and R. J. Full, Proc. Natl. Acad. Sci. U. S. A., 2002, 99, 12252-12256.

3 R. Spolenak, S. Gorb, H. J. Gao and E. Arzt, Proc. R. Soc. London, Ser. A, 2005, 461, 305-319.

4 M. Varenberg, A. Peressadko, S. Gorb and E. Arzt, Appl. Phys. Lett., 2006, 89, 121905.

5 A. V. Spuskanyuk, R. M. McMeeking, V. S. Deshpande and E. Arzt, Acta Biomater., 2008, 4, 1669-1676.

6 A. del Campo, C. Greiner and E. Arzt, Langmuir, 2007, 23, 10235 10243.

7 M. Varenberg, N. M. Pugno and S. N. Gorb, Soft Matter, 2010, 6, 3269-3272.

8 S. Gorb, M. Varenberg, A. Peressadko and J. Tuma, J. R. Soc. Interface, 2007, 4, 271-275.

9 E. Arzt, S. Gorb and R. Spolenak, Proc. Natl. Acad. Sci. U. S. A., 2003, 100, 10603-10606.

10 Y. Tian, N. Pesika, H. B. Zeng, K. Rosenberg, B. X. Zhao, P. McGuiggan, K. Autumn and J. Israelachvili, Proc. Natl. Acad. Sci. U. S. A., 2006, 103, 19320-19325.

11 M. Varenberg and S. Gorb, J. R. Soc. Interface, 2007, 4, 721-725.

12 M. Varenberg and S. Gorb, J. R. Soc. Interface, 2008, 5, 785-789.

13 L. Heepe, M. Varenberg, Y. Itovich and S. N. Gorb, J. R. Soc. Interface, 2011, 8, 585-589.

14 M. P. Murphy, B. Aksak and M. Sitti, Small, 2009, 5, 170-175.

15 K. Autumn, A. Dittmore, D. Santos, M. Spenko and M. Cutkosky, J. Exp. Biol., 2006, 209, 3569-3579.

16 N. E. Stork, Zoological Journal of the Linnean Society, 1980, 68, 173306.

17 H. J. Gao and S. H. Chen, J. Appl. Mech., 2005, 72, 732-737.

18 Z. L. Peng, S. H. Chen and A. K. Soh, Int. J. Solids Struct., 2010, 47, 1952-1960.

19 B. N. J. Persson and S. Gorb, J. Chem. Phys., 2003, 119, 11437-11444.

20 N. S. Pesika, Y. Tian, B. X. Zhao, K. Rosenberg, H. B. Zeng, P. McGuiggan, K. Autumn and J. N. Israelachvili, J. Adhes., 2007, 83, 383-401.

21 Z. L. Peng and S. H. Chen, Phys. Rev. E: Stat., Nonlinear, Soft Matter Phys., 2011, 83, 051915.

22 Z. L. Peng and S. H. Chen, Colloids Surf., B, 2011, 88, 717-721.

23 G. Huber, S. N. Gorb, R. Spolenak and E. Arzt, Biol. Lett., 2005, 1, 2-4.

24 G. L. Li and T. C. Chang, Acta Mech. Solida Sin., 2011, 24, 318-325.

25 G. Carbone, E. Pierro and S. N. Gorb, Soft Matter, 2011, 7, 55455552.

26 J. C. Simo, F. Armero and R. L. Taylor, Comput. Methods Appl. Mech. Eng., 1993, 110, 359-386.

27 V. Tvergaard and J. W. Hutchinson, Int. J. Solids Struct., 1996, 33, 3297-3308.

28 P. Rahulkumar, A. Jagota, S. J. Bennison and S. Saigal, Int. J. Solids Struct., 2000, 37, 1873-1897.

29 J. H. Rose, J. Ferrante and J. R. Smith, Phys. Rev. Lett., 1981, 47, 675-678.

30 J. R. Willis, J. Mech. Phys. Solids, 1967, 15, 151-162.

31 X. P. Xu and A. Needleman, J. Mech. Phys. Solids, 1994, 42, $1397-$ 1434.

32 H. J. Gao, X. Wang, H. M. Yao, S. Gorb and E. Arzt, Mech. Mater., 2005, 37, 275-285.

33 S. N. Gorb and M. Varenberg, J. Adhes. Sci. Technol., 2007, 21, 1175 1183.

34 C. Y. Hui, N. J. Glassmaker, T. Tang and A. Jagota, J. R. Soc. Interface, 2004, 1, 35-48.

35 M. Varenberg, B. Murarash, Y. Kligerman and S. N. Gorb, Appl. Phys. A: Mater. Sci. Process., 2011, 103, 933-938. 